\section{Billets d'humour} et d'humeur

\section{Que ferez-vous le 1er avril 2006?}

«Je vais à la manifestation à Berne!» devrait répondre tout médecin exerçant dans ce pays, sans la moindre hésitation! Le but n'est bien sûr pas de vociférer «houleusement» contre notre ministre (même s'il le méritait...) ni d'organiser une grande foire des revendications. Nous ne voulons pas manifester «contre» tout ce qui nous est imposé, mais bien «pour» une médecine de qualité, que nous sommes seuls à savoir définir.

Certes, on peut se poser la question de savoir si une manifestation est la meilleure réponse aux «couchepineries» introduites à l'occasion de la Nouvelle année, et s'il n'aurait pas été judicieux d'associer aussi les spécialistes à cette démarche. Là n'est pas vraiment la question, les médecins de premier recours étant les plus menacés ce sont eux qui ont pris l'initiative et c'est très bien ainsi.

Ce que chaque médecin, généraliste ou spécialiste, doit réaliser sans tarder, c'est que nous sommes tous concernés et que les mesures qui attaquent le laboratoire et imposent la prescription de génériques, sans parler du scandale de la radiologie, ne sont que des ballons d'essai, pour tester notre capacité à réagir et à nous défendre. Sans signe clair de notre part, les mesures de contraintes de toutes sortes vont pleuvoir sur l'ensemble du corps médical, et l'exercice de notre profession deviendra pratiquement impossible en tant qu'indépendant.

La présence de milliers de médecins le 1er avril, qui se réunissent pour dire leur envie de travailler dans des conditions décentes, de pouvoir gérer leur cabinet de manière à offrir les meilleurs soins aux patients dans un respect des coûts justes de leur travail doit être un signal extrêmement fort vis-à-vis du monde politique et de la direction de la FMH. Nous devons exprimer haut et fort notre volonté de continuer à offrir une médecine de qualité basée sur des soins de premier recours de qualité et démontrer notre détermination essentiellement face à deux personnes: d'une part Monsieur Couchepin qui doit sentir, à travers notre présence en nombre, la force de notre conviction, et d'autre part notre Président, le Dr Jacques de Haller, qui a aussi besoin de signaux forts de la base pour mener à bien la politique de la FMH.

En fait, les généralistes ont pris un gros risque en annonçant dès maintenant la manifestation dans la presse. Car, sur ce coup-là, les médecins n'ont pas droit à l'erreur! Nous ne pouvons pas imaginer le cauchemar de la vision de 200 ou 300 personnes disséminées sur la Place Fédérale... Cela représenterait un coup terrible et probablement fatal pour les médecins de Suisse, la preuve qu'attend Monsieur Couchepin que les médecins sont incapables de se fédérer autour d'une idée et qui reviendrait à l'encourager dans ces décisions non concertées qui nous gâchent chaque jour un peu plus le plaisir de travailler. Ce serait surtout un signal terriblement démotivant pour notre Président, qui pourrait le conduire à penser qu'il ne vaut guère la peine de se fatiguer à la tâche pour des gens qui sont incapables de consacrer quelques heures à la défense de leur outil de travail. Peut-on imaginer le climat des négociations Couchepin - de Haller (s'il y en avait encore...!) si la manifestation venait à être un échec?

Réfléchissez-y bien, Chers Confrères, venez nombreux, soyez des nôtres, votre présence est indispensable car personne n'ira à votre place! C'est une occasion unique de démontrer notre volonté et notre solidarité, il y va de notre crédibilité !

Dr Jean-Pierre Grillet

Dermatologie et Allergologie FMH

CH-1206 Genève 